\title{
On the extended 23rd solar cycle
}

\author{
Valery N. Krivodubskij \\ Astronomical Observatory of Kyiv National Taras Shevchenko University \\ Observatorna Street 3, Kyiv - 53, 04053, Ukraine \\ email: krivod1@observ.univ.kiev.ua
}

\begin{abstract}
An explanation of the mystery of the extended 23rd solar cycle duration about 13 years in the frame of non-linear regime of the $\alpha \Omega$ - dynamo model is proposed. The calculated dynamo-period of the solar cycle, $T$, depends (in the inverse proportion) on the intensity of the $\alpha$ - effect in the solar convection zone (SCZ). As well, the intensity of the $\alpha$ - effect in non-linear regime depends (also in the inverse proportion) on the value of toroidal magnetic field, $B_{T}$ (magnetic alpha-quenching). Thus, the calculated period is in direct proportion to the value of toroidal magnetic field: the stronger toroidal field $B_{T}$ in certain cycle, the longer dynamo-period $T$ of this cycle. Since the toroidal field is hidden in the deep layers of the SCZ, it is necessary to know some other magnetic experimental evidence that reflects something like information about inner toroidal field. In this connection we allow for that the strong toroidal field is transported by magnetic buoyancy to the solar surface and produces here the sunspots, so they carry indirect information on $B_{T}$. In this connection we took into account up-to-date observed data on the essential increase of the averaged annual module of the magnetic field of the large-scale sunspots, $B_{s p}$, in the $23 \mathrm{rd}$ cycle; and then we made calculation of the alpha-quenching which depends on these referred data. It is important to know only relative variations of magnetic index $B_{s p}$ for calculation of the dynamo-period variation. Our estimations showed that the average solar period, which is about 11 years, must increase by a factor of 1,2 ; so the calculated 23rd cycle dynamo-period would be about 13 years.
\end{abstract}

Keywords. Sun: activity, Sun: sunspots, magnetic fields

According to mechanism of the solar $\alpha \Omega$ - dynamo, a strong toroidal magnetic field, $B_{T}$, is generated in the SCZ by differential rotation stretching a weak poloidal magnetic field, $B_{P}$, (the $\Omega$ - effect), while the helical turbulent convection regenerates a new poloidal field, with the magnetic polarity opposite to that of previous cycle, by twisting the toroidal field-lines (the $\alpha$ - effect). The period of the dynamo-cycle is determined by equation

$$
T=\frac{2 \pi}{\left[\frac{1}{2}\left|\alpha\left(B_{T}\right) \partial \Omega / \partial r\right|\right]^{1 / 2}}
$$

where $\alpha$ is the parameter of mean helicity of turbulent convective pulsations, $\partial \Omega / \partial r$ is the radial gradient of the angle velocity, $\Omega$, in the SCZ. It is obvious, that the calculated period of the dynamo-cycle $T$ is reducing when the $\alpha$-parameter is increasing, and, viceversa, reducing of the $\alpha$ - parameter leads to extending of the period $T$. In non-linear regime $\alpha$-parameter depends, in the inverse proportion, on the value of toroidal filed $B_{T}$ : magnetic alpha-quenching (Rüdiger \& Kitchatinov 1993; Krivodubskij 2005). Then the calculated period is in direct proportion to the toroidal magnetic field value: the stronger toroidal field $B_{T}$ in cycle, the longer calculated dynamo-period $T$.

However the toroidal field is hidden in the deep layers of the SCZ. So its value that necessary for the calculation of the alpha-quenching cannot be directly obtained from the observations. At the same time it is known that the strong toroidal field is transported 


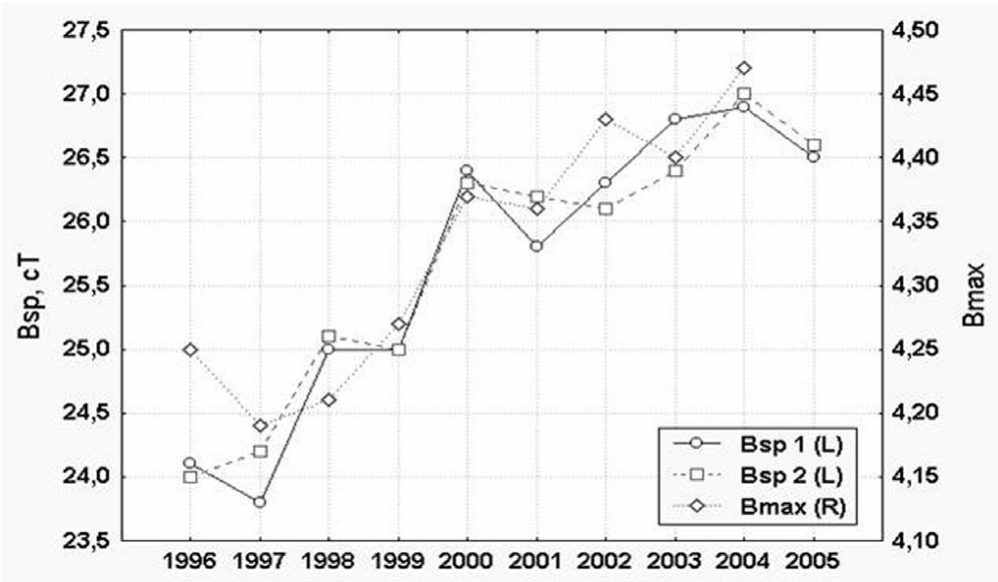

Figure 1. Increasing of the sunspots magnetic fields in the 23rd solar cycle (about 2,000 visual measurements). Index $B_{s p} 1(\mathrm{~L})$ is the observed annual averaged module of the magnetic fields (centiTesla) of the large-scale sunspots, 30-60 arcsec (courtesy of Lozitskaja et al. 2007). Only index $B_{s p} 1(\mathrm{~L})$ (left ordinate axis) was used in this paper for the alpha-quenching calculation.

by magnetic buoyancy to the solar surface and produces here the sunspots. Therefore the sunspots magnetic fields provide something like information about the values of the deep toroidal field. In this connection we hope that some sunspots magnetic parameters will help us to estimate the alpha-quenching. Note that it is necessary to know only relative variation of the sunspots parameters for the calculation of the dynamo-period variation.

Recently Lozitska (2005) proposed a new index of the annual averaged module of the magnetic fields for the sunspots with penumbra diameter 30-60 arcsec $(22-44 \mathrm{Mm})$, $B_{s p}$, measured by visual method on the Zeeman splitting in the Fe I $\lambda \lambda 525.02$ and $630.25 \mathrm{~nm}$ lines. We suppose that this magnetic index satisfactory reflects the intensity of the hidden inner toroidal field $B_{T}$. Later Lozitskaja et al. (2007), according to study about 2,000 measurements at four observatories (Astronomical Observatory of Kyiv National University, Crimean Astrophysical Observatory, Astronomical Observatory of Ural State University and Mount Wilson Observatory), discovered that magnetic index $B_{s p}$ was increased on $\approx 13 \%$ at the maximum of the 23rd cycle as against the cycle minimum (Fig. 1). We used these up-to-date observed data of the essential increasing of index $B_{s p}$ for the alpha-quenching calculation. The magnetic back reaction of increasing fields $B_{s p}$ on helical turbulent convection, self-induction, causes suppression (quenching) of the $\alpha$ effect by a factor about 0,7 at the maximum cycle. Under this condition the dynamoperiod of the 23rd cycle is bound to prolong by a factor of 1,2 in comparison with previous cycles. Thus, with the assumption that the average solar cycle duration is about 11 year, the calculated 23rd cycle dynamo-period to be about 13 years. One more possibility for the explanation of the extended 23rd cycle is taking into account the perturbations of the meridional flow during the cycle (will be study in our future researches).

\section{References}

Krivodubskij, V. N. 2005, AN, 3, 61

Lozitska, N. I. 2005, Kinenatika i Fizika Nebes. Tel (Suppl.), 5, 151

Lozitskaja, N. I., Liamova, G. V., \& Malashchuk, V. M. 2007, Izvestija CrAO, 107, No 4, 59

Rüdiger, G. \& Kitchatinov, L. L. 1993, AA, 269, 581 